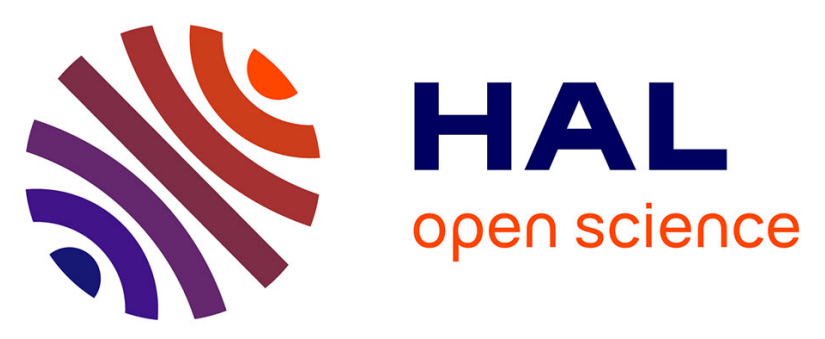

\title{
Automated closed-loop versus standard manual oxygen administration after major abdominal or thoracic surgery: an international multicentre randomised controlled study
}

Erwan L'Her, Samir Jaber, Daniel Verzilli, Christophe Jacob, Brigitte Huiban, Emmanuel Futier, Thomas Kerforne, Victoire Pateau, Pierre-Alexandre Bouchard, Maëlys Consigny, et al.

\section{- To cite this version:}

Erwan L'Her, Samir Jaber, Daniel Verzilli, Christophe Jacob, Brigitte Huiban, et al.. Automated closed-loop versus standard manual oxygen administration after major abdominal or thoracic surgery: an international multicentre randomised controlled study. European Respiratory Journal, In press, pp.2000182. 10.1183/13993003.00182-2020 . hal-02924879

\author{
HAL Id: hal-02924879 \\ https://hal.science/hal-02924879
}

Submitted on 16 Jun 2021

HAL is a multi-disciplinary open access archive for the deposit and dissemination of scientific research documents, whether they are published or not. The documents may come from teaching and research institutions in France or abroad, or from public or private research centers.
L'archive ouverte pluridisciplinaire HAL, est destinée au dépôt et à la diffusion de documents scientifiques de niveau recherche, publiés ou non, émanant des établissements d'enseignement et de recherche français ou étrangers, des laboratoires publics ou privés. 


\title{
Automated closed-loop versus standard manual oxygen administration after major abdominal or thoracic surgery: an international multicentre randomised controlled study
}

\author{
Erwan L'Her ${ }^{1,2}$, Samir Jaber ${ }^{3}$, Daniel Verzilli ${ }^{3}$, Christophe Jacob ${ }^{4}$, \\ Brigitte Huiban ${ }^{4}$, Emmanuel Futier $^{5}$, Thomas Kerforne ${ }^{6}$, Victoire Pateau ${ }^{2,7}$, \\ Pierre-Alexandre Bouchard ${ }^{8}$, Maëlys Consigny ${ }^{9}$ and François Lellouche ${ }^{8}$
}

\begin{abstract}
Introduction: Hypoxaemia and hyperoxaemia may occur after surgery, with related complications. This multicentre randomised trial evaluated the impact of automated closed-loop oxygen administration after high-risk abdominal or thoracic surgeries in terms of optimising the oxygen saturation measured by pulse oximetry time within target range.

Methods: After extubation, patients with an intermediate to high risk of post-operative pulmonary complications were randomised to "standard" or "automated" closed-loop oxygen administration. The primary outcome was the percentage of time within the oxygenation range, during a 3-day frame. The secondary outcomes were the time with hypoxaemia and hyperoxaemia under oxygen.

Results: Among the 200 patients, time within range was higher in the automated group, both initially ( $\leqslant 3 \mathrm{~h}$; $91.4 \pm 13.7 \%$ versus $40.2 \pm 35.1 \%$ of time, difference $+51.0 \%$ ( $95 \%$ CI $-42.8-59.2 \%) ; \mathrm{p}<0.0001)$ and during the 3 -day period $(94.0 \pm 11.3 \%$ versus $62.1 \pm 23.3 \%$ of time, difference $+31.9 \%$ (95\% CI $26.3-37.4 \%$ ); $\mathrm{p}<0.0001$ ). Periods of hypoxaemia were reduced in the automated group ( $\leqslant 3$ days; $32.6 \pm 57.8 \mathrm{~min}(1.2 \pm 1.9 \%)$ versus $370.5 \pm 594.3 \mathrm{~min}(5.0 \pm 11.2 \%)$, difference $-10.2 \%$ (95\% CI $-13.9--6.6 \%) ; \mathrm{p}<0.0001)$, as well as hyperoxaemia under oxygen ( $\leqslant 3$ days; $5.1 \pm 10.9 \mathrm{~min}(4.8 \pm 11.2 \%)$ versus $177.9 \pm 277.2 \mathrm{~min}(27.0 \pm 23.8 \%)$, difference $-22.0 \%$ (95\% CI $-27.6--16.4 \%)$; $<<0.0001)$. Kaplan-Meier analysis depicted a significant difference in terms of hypoxaemia $(\mathrm{p}=0.01)$ and severe hypoxaemia $(\mathrm{p}=0.0003)$ occurrence between groups in favour of the automated group. 25 patients experienced hypoxaemia for $>10 \%$ of the entire monitoring time during the 3 days within the standard group, as compared to the automated group $(\mathrm{p}<0.0001)$.

Conclusion: Automated closed-loop oxygen administration promotes greater time within the oxygenation target, as compared to standard manual administration, thus reducing the occurrence of hypoxaemia and hyperoxaemia.
\end{abstract}

This article has supplementary material available from erj.ersjournals.com

This study is registered at Clinicaltrials.gov with identifier NCT02546830.

Data availability: Study protocol, SAP, informed consent, and study report will be available to anyone who wishes to access the data on individual demand to the promoter. 


\section{Introduction}

Current standards for prescribing oxygen recommend providing adequate flows to correct hypoxaemia and avoid hyperoxaemia $[1,2]$. While the deleterious effects of hypoxaemia are well known, the potential harmful effects of hyperoxaemia are underappreciated; hyperoxaemia may increase mortality in severe COPD patients [3-5] and may cause cardiac and neurological adverse toxicities in certain situations [6-8]. Precise control of oxygen $\left(\mathrm{O}_{2}\right)$ flows is difficult to achieve in clinical practice and is time-consuming [9]; Oxygen therapy is thus commonly administered with poor adherence to treatment recommendations.

The $\mathrm{FreeO}_{2}$ system is an innovative device, developed in collaboration between researchers at university hospitals in Brest (France) and Québec (Canada) and Oxynov Inc., an R\&D spin-off from Laval University in Québec. FreeO $\mathrm{O}_{2}$ is a closed-loop device that automates oxygen administration to spontaneously breathing patients, in response to continuous pulse oximetry $\left(\mathrm{S}_{\mathrm{pO}_{2}}\right)$ measurements [10]. Automated $\mathrm{O}_{2}$ administration can maintain constant $S_{\mathrm{pO}_{2}}$, within a pre-determined range using variable $\mathrm{O}_{2}$ flows, as opposed to manual oxygen administration where the flow is kept constant, with variable $S_{\mathrm{pO}_{2}}$ values. In preterm infants receiving mechanical ventilation, automated oxygen control results in more time spent within the intended $\mathrm{S}_{\mathrm{pO}_{2}}$ target [11-13]. In healthy adults with induced hypoxaemia, such a system was more efficient to maintain $S_{\mathrm{pO}_{2}}$ within the oxygenation target, while ensuring a significant reduction in hypoxaemia and hyperoxaemia periods, as compared to constant $\mathrm{O}_{2}$ flows [14]. Its efficacy in terms of optimising the $S_{\mathrm{pO}_{2}}$ time within target range has also been validated in hospitalised COPD patients [15], or during the early emergency care of patients with acute respiratory distress [10].

Following major abdominal or thoracic surgery, the risk of hypoxaemia may be high while considering patient clinical status (obstructive sleep apnoea, restrictive pathologies related to obesity, frequent comorbidities), the type of surgery and anaesthesia, and the fact that patients are frequently given opioid treatment post-operatively [16-22]. The prevalence of post-operative hypoxaemia is often underestimated, and there remains a significant number of patients who still develop respiratory complications following extubation, thereby suggesting that there is room for improvement in these patients. Oxygen therapy is almost invariably applied after elective extubation using low-flow devices to correct oxygenation impairment, but may not always prevent the post-operative deterioration in respiratory function. Hypoxaemia may occur either during the immediate post-operative period (it is therefore mainly related to surgery or anaesthesia) or may be delayed up to 3 days without a clear trigger or underlying pathology. If the arterial oxygen tension is increased excessively, it may also lead to hyperoxaemia-mediated vasoconstriction in almost all vascular beds, including coronary arteries, and promote atelectasis formation [23].

The potential interest of the $\mathrm{FreeO}_{2}$ system, using artificial intelligence, closed-loop adjustments and predictive analytics, is 1) to perform rapid $\mathrm{O}_{2}$ adjustments in response to oxygenation condition variations (up to each second), or to any physiological condition changes (movement, speech, eating ...); 2) to enable remote monitoring and continuous data recording in isolated clinical settings (i.e. non-intensive care unit (ICU) surgical ward) in order to detect at a very early stage clinical deterioration through the integration and fusion of information; and 3) to avoid the maintenance of unnecessarily high $\mathrm{O}_{2}$ flow that may be deleterious.

The $\mathrm{FreeO}_{2}$ Post-Op trial aimed to evaluate the clinical impact of automated closed-loop versus standard manual oxygen administration in terms of oxygenation, after major abdominal or thoracic surgeries. We hypothesised that automated oxygen administration would promote better adherence to clinical guidelines than conventional therapy, thus optimising the $S_{\mathrm{pO}_{2}}$ time within the target range for up to 3 days following surgery.

\section{Methods}

Study design

The $\mathrm{FreeO}_{2}$ PostOp international, multicentre, randomised, controlled trial was funded by the French ministry of health (Programme Hospitalier de Recherche Clinique Interrégional HUGO 2012-199) and promoted by Brest University Hospital. The trial was overseen by a steering committee and safety

Affiliations: ${ }^{1}$ Medical Intensive Care, CHRU de Brest - La Cavale Blanche, Brest, France. ${ }^{2}$ LATIM INSERM UMR 1101, FHU Techsan, Université de Bretagne Occidentale, Brest, France. ${ }^{3}$ Intensive Care Unit, Dept of Anaesthesiology B, DAR B CHU de Montpellier, Hôpital Saint Eloi, Université Montpellier 1, Montpellier, France. ${ }^{4}$ Anaesthesiology Dept, CHRU de Brest - La Cavale Blanche, Brest, France. ${ }^{5}$ Anaesthesiology Dept, Hôpital Estaing, Centre Hospitalier Universitaire Clermont-Ferrand, Clermont-Ferrand, France. ${ }^{6}$ Anaesthesiology Dept, CHU de Poitiers, Cedex, France. ${ }^{7}$ Oxynov Inc., Technopôle Brest Iroise, Plouzané, France. ${ }^{8}$ Centre de recherche de l'Institut de Cardiologie et de Pneumologie de Québec, Quebec, Canada. ${ }^{9}$ Centre d'Investigation Clinique CIC INSERM 1412, CHRU Brest - La Cavale Blanche, Brest, France.

Correspondence: Erwan L'Her, Médecine Intensive et Réanimation, CHRU de Brest - La Cavale Blanche, Bvd Tanguy-Prigent, 29609, Brest Cedex, France. E-mail: Erwan.lherlachu-brest.fr 
monitoring board, composed of three independent experts. The institutional review board of Brest University Hospital approved the trial for all French centres, and the institutional review board from the Québec Heart and Lung Institute (Canada) approved the trial for their own centre. The FreeO 2 Post-Op study was conducted in accordance with the declaration of Helsinki and registered on September 11, 2015 at the Agence Nationale de Sécurité du Médicament et des Produits de Santé (ID RCB 2014-A00615-42) and Clinicaltrials.gov (NCT02546830).

The $\mathrm{FreeO}_{2}$ PostOp study took place at five university hospitals in France and Canada (Brest, Clermont-Ferrand, Montpellier, Poitiers, Québec). The first patient inclusion was performed in January 2016 and the last inclusion was in September 2018. All centres were monitored regularly to check adherence to the protocol and accuracy of the recorded data. An investigator at each centre was responsible for enrolling patients and ensuring adherence to the protocol. Research assistants were responsible for patient follow-up and for completing the electronic case report form. Although assignment to the study could not be blinded, all clinicians in charge of the patients remained unaware of the study outcomes, i.e. while clinicians were aware of the $\mathrm{O}_{2}$ flow and $\mathrm{S}_{\mathrm{pO}_{2}}$ values modifications (in the automated group, simply while looking at the device's screen), they were not constantly looking at the device during the 3-day monitoring frame, and thus could not be aware of all $\mathrm{O}_{2}$ flow modifications and $S_{\mathrm{PO}_{2}}$ time within the target range in both groups over time.

\section{Patients}

To be eligible, adult patients (aged $\geqslant 18$ years) were to be screened for scheduled abdominal or thoracic surgery during the anaesthesia consultation and to be considered as requiring general anaesthesia with an expected duration of $\geqslant 2 \mathrm{~h}$ in the participating centres. Patients had to present an intermediate to high risk of post-operative pulmonary complications, with an ARISCAT (Assess Respiratory Risk in Surgical Patients in Catalonia) risk score $\geqslant 26[24]$.

Informed consent was signed by the patient before the surgery. Figure 1 shows the flow chart of the $\mathrm{FreeO}_{2}$ PostOp study.

Patients fulfilling one or more of the following criteria were excluded, to provide a relatively homogenous study population and avoid potential confounding factors in the interpretation: patients with a body mass index $\geqslant 35 \mathrm{~kg} \cdot \mathrm{m}^{-2}$, patients with obstructive sleep apnoea and pregnant patients.

\section{Randomisation and masking}

Patients were randomised after surgery if they fulfilled all the following criteria: availability of the $\mathrm{FreeO}_{2}$ prototype; absence of criteria of severity justifying the immediate utilisation of ventilatory support (loss of consciousness with a Glasgow Coma Score $\leqslant 12$, serious ventricular rhythm disorders, haemodynamic instability (systolic blood pressure $<80 \mathrm{mmHg}$ or recourse to vasopressors), cardiac or respiratory arrest, $\mathrm{pH}<7.35$ and arterial carbon dioxide tension $\left(\mathrm{P}_{\mathrm{aCO}_{2}}\right)>55 \mathrm{mmHg}$ (if measured) or necessity for oxygen flow $<15 \mathrm{~L} \cdot \mathrm{min}^{-1}$ to maintain a $S_{\mathrm{pO}_{2}}>92 \%$ ); no emergent surgery required for an adverse event; and available pulse oximetry signal.

Computer-generated randomisation was performed within a maximal 1-h delay following endotracheal extubation in the recovery room. It was performed using random blocks in a 1:1 ratio, with the use of a centralised web-based management system (Clinfile; MultiHealth, Velisy Villacoublay, France). Patients were assigned to standard manual oxygen administration ("standard") or to automated closed-loop oxygen administration ("automated"). Stratification was performed according to the study centre and medical history of COPD. After randomisation, treatment was initiated within $1 \mathrm{~h}$.

Although the individual study assignments of the patients were not masked, the coordinating centre and all the investigators remained unaware of the study group outcomes until the data were locked. Before locking the database and after trial completion, the review board checked data and decided which patients could be included in the intention-to-treat analysis in accordance with good clinical practice guidelines. An adjudication committee, who was unaware of the study groups, extracted pulse oximetry curves and values and reviewed all the data to analyse the events occurring during the procedure in order to verify patient safety. The complete methodology of the study has been published previously [25].

\section{Procedures}

The maximal study participation was 3 days. The study was stopped before 3 days if the patient was to be discharged from hospital earlier.

Automated $\mathrm{O}_{2}$ was administered using the FreeO ${ }_{2}$ system (Oxynov Inc., Québec, QC, Canada) which was set to maintain $S_{\mathrm{pO}_{2}}$ at $92-96 \%$ for non-COPD patients or $88-92 \%$ for COPD patients, as recommended by international guidelines $[1,2]$. FreeO $\mathrm{O}_{2}$ is equipped with a $S_{\mathrm{pO}_{2}}$ monitor and an electronically controlled 
valve that automatically adjusts oxygen flows from 0 to $20 \mathrm{~L} \cdot \mathrm{min}^{-1}$ on a per second basis, with a precision of $0.1 \mathrm{~L} \cdot \mathrm{min}^{-1}$, according to a closed-loop algorithm, in order to reach the predetermined $\mathrm{S}_{\mathrm{pO}_{2}}$ target [14]. Standard oxygen was administered using manual flowmeters, according to standard clinical procedures. All participating units were encouraged to use the same standardised $S_{\mathrm{pO}_{2}}$ target. In both arms, oxygen was administered either using nasal prongs for low flow $\left(\mathrm{O}_{2}<6 \mathrm{~L} \cdot \mathrm{min}^{-1}\right)$ in case of clinical stability, or an open face mask for low or medium flow. On a routine basis, we advocated the use of face masks for all patients, in order to be able to deliver either low to medium flow, or transient increases in response to patient needs, especially in response to physical effort.

To accurately determine the incidence and severity of post-operative $S_{\mathrm{pO}_{2}}$ variations, continuous oximetry recordings were performed in each group during the entire study, using the $\mathrm{FreeO}_{2}$ monitoring system at $1 \mathrm{~Hz}$ (one $S_{\mathrm{pO}_{2}}$ value per second) and connected to Nonin 6000 CA flexible adult single-use digital sensors (Nonin, Plymouth, MN, USA). The position of the sensors was checked at least every $12 \mathrm{~h}$.

Considering the variety of pathological cases for patients requiring surgery, medical treatment (i.e. fluid administration, prophylactic antibiotics and post-operative pain management), including respiratory support, was determined by the attending physicians based on a clinical needs assessment. Medical diagnoses associated with general characteristics (e.g. COPD, cardiopathy) were determined during the anaesthesia consultation, according to patient medical files. According to the potential risk of pulmonary complications (ARISCAT score), specific attention was paid to this point of interest.

\section{Measurements and outcomes}

The primary outcome measure was the percentage of time spent within the $S_{\mathrm{pO}_{2}}$ target zone, during a 3 -day time frame. While continuous oximetry was recorded in both groups at a frequency of $1 \mathrm{~Hz}$, even short episodes of oxygen desaturation were detected. The target zone of oxygen saturation was $S_{\mathrm{pO}_{2}} 88-$ $92 \%$ for COPD and $92-96 \%$ for non-COPD patients; this target zone was considered for patients receiving

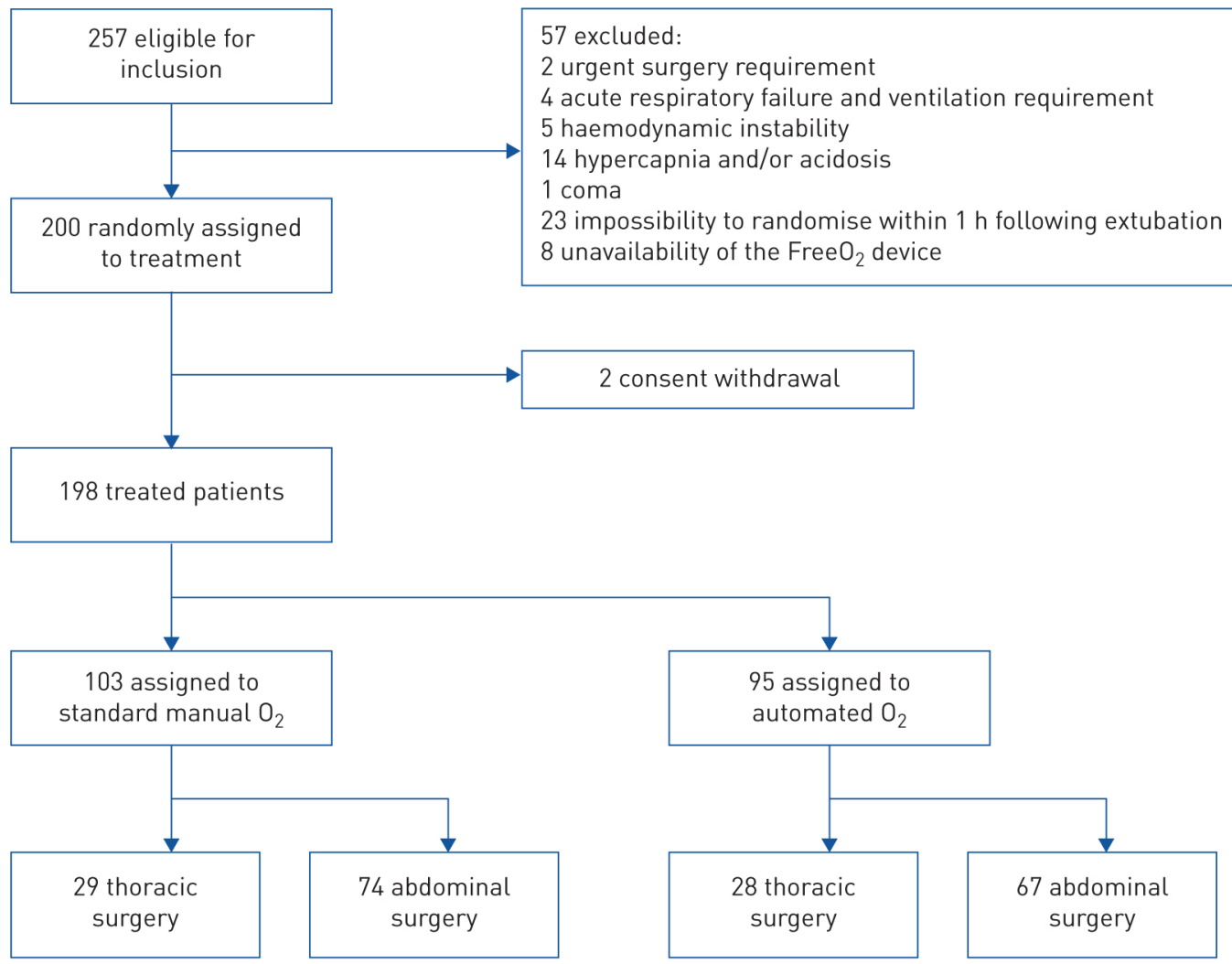

FIGURE 1 Flow chart of the $\mathrm{FreeO}_{2}$ PostOp study. Inclusion was performed during the anaesthesia consultation, and randomisation was performed not later than $1 \mathrm{~h}$ following extubation in the recovery room. For non-COPD patients, the target was an oxygen $\left(\mathrm{O}_{2}\right)$ saturation measured by pulse oximetry $\left(\mathrm{S}_{\mathrm{pO}_{2}}\right)$ range of 92-96\%; for COPD patients, the target range was $88-92 \%$. Patients were assigned either to standard manual oxygen administration ("standard"), or automated closed-loop oxygen administration ("automated"); in both groups, continuous $\mathrm{S}_{\mathrm{pO}_{2}}$ recording was performed for up to 3 days according to the $\mathrm{FreeO}_{2}$ system. Clinical data were recorded each hour during the first $3 \mathrm{~h}$ of care and twice daily for up to 3 days. 
additional oxygen; patients without oxygen, but with values higher than the range, were considered adequately treated.

Several secondary outcome measures were evaluated during a 3-day time frame: nursing workload assessed by the number of manual oxygen flow adjustments; time with hypoxaemia $\left(S_{\mathrm{pO}_{2}}<88 \%\right.$ for COPD and $<92 \%$ for non-COPD), severe hypoxaemia $\left(S_{\mathrm{pO}_{2}}<85 \%\right.$ for all $)$; hyperoxaemia under oxygen $\left(S_{\mathrm{pO}_{2}}>98 \%\right)$, and the first occurrence of a clinically significant hypoxaemia and severe hypoxaemia event, defined as the occurrence of such an event for $\geqslant 30 \mathrm{~s}$.

Other outcome measures were assessed during a maximal 28-day time frame: duration of oxygen administration during hospitalisation, number of complications related to oxygen administration, frequency of use of ventilation (invasive or non-invasive), duration of hospitalisation and survival rate.

\section{Statistical methods}

Based on previous studies, we estimated $85 \%$ of time within the oxygenation range with the automated closed-loop oxygen administration system $\left(\mathrm{FreeO}_{2}\right)$ and a standard deviation $\geqslant 30 \%$. Thus, a total number of 200 patients was needed to demonstrate a 15\% decrease in the absolute difference between the standard and $\mathrm{FreeO}_{2}$ (automated) groups.

All analyses were performed by an independent statistician, on an intention-to-treat basis using SAS V.9.4 statistical software (SAS Institute, Cary, NC, USA). Continuous variables were analysed using standard parameters (median, interquartile ranges and extreme values, or mean $\pm \mathrm{SD}$ ), while categorical variables were analysed in the form of absolute frequency and percentage. The main criteria of evaluation (percentage of time within the considered $S_{\mathrm{pO}_{2}}$ range) was compared between the two groups by means of variance analysis, according to stratification. Secondary criteria of evaluation were compared between the two treatment groups by means of the t-test (or the Mann-Whitney U-test, if necessary) for continuous quantitative variables and the Chi-squared test (or Fisher's exact test) for qualitative variables. The probability of a significant hypoxaemia episode occurring within groups was compared using the KaplanMeier estimate and the log-rank test. A two-tailed p-value $\leqslant 0.05$ was considered statistically significant.

\section{Results}

Patients

The time course of patient participation is detailed in figure S1. 200 patients were randomised to either the standard $(n=103)$ or the automated group $(n=97)$ (figure 1$)$. No patients were excluded solely due to unavailable pulse oximetry signal, but rather to deterioration of medical condition. Patient demographics, physiological characteristics and comorbidities were well balanced between the groups (table 1). Principal outcome parameters could be measured in only 169 (84.5\%) patients due to recording problems and $S_{\mathrm{pO}_{2}}$ signal loss over the time frame ( 83.5 and $85.6 \%$ in the standard and automated groups, respectively).

\section{Oxygenation parameters and primary outcome}

Oxygenation parameters results are provided in table 2 , for the short-term $(\leqslant 3 \mathrm{~h})$ and the long-term ( $\leqslant 3$ days) periods (see also supplementary figures S2 and S3 for illustration). Time within range was higher in the automated group, either during the short-term ( $\leqslant 3 \mathrm{~h} ; 91.4 \pm 13.7 \%$ versus $40.2 \pm 35.1 \%$ of time; adjusted difference $+51.0 \%$, 95\% CI $-42.8-59.2 \% ; \mathrm{p}<0.0001)$ or the long-term period ( $\leqslant 3$ days; $94.0 \pm 11.3 \%$ versus $62.1 \pm 23.3 \%$ of time; adjusted difference $+31.9 \%, 95 \%$ CI $26.3-37.4 \%$; $<<0.0001$ ). Periods of hypoxaemia were reduced in the automated group ( $\leqslant 3$ days; $32.6 \pm 57.8 \mathrm{~min}(1.2 \pm 1.9 \%)$ versus $370.5 \pm 594.3 \mathrm{~min}(5.0 \pm 11.2 \%)$; adjusted difference $-10.2 \%$, 95\% CI $-13.9--6.6 \%$; $<0.0001$ ), as well as hyperoxaemia under oxygen ( $\leqslant 3$ days; $5.1 \pm 10.9 \min (4.8 \pm 11.2 \%)$ versus $177.9 \pm 277.2 \mathrm{~min}(27.0 \pm 23.8 \%)$; adjusted difference $-22.0 \%, 95 \%$ CI $-27.6--16.4 \%$; $<<0.0001)$.

In the standard group, up to 25 patients experienced hypoxaemia for $>10 \%$ of the time during the 3 days of recording, while this adverse event was not observed for patients assigned to the automated group $(\mathrm{p}<0.0001)$ (table 3).

A significant difference in terms of hypoxaemia $(\mathrm{p}=0.01)$ and severe hypoxaemia $(\mathrm{p}=0.0003)$ occurrence was observed between groups, always in favour of the automated group (figure 2).

\section{Secondary outcomes}

Secondary outcomes results are detailed in table 3.

Mean oxygen flow was not different in between groups, but flow variations $>50 \%$ were more frequent in the automated group ( $39 \%$ versus $19.5 \%$ of patients; $\mathrm{p}<0.001$ ), as well as weaning in the recovery room ( $14.1 \%$ versus $4.3 \%$ of patients; $\mathrm{p}<0.001)$ (table 2 ). Oxygen was still prescribed after day 3 in $34(33.3 \%)$ versus $17(18.3 \%)$ patients in the standard and automated groups, respectively $(p=0.01)$. 
No difference was observed in between groups, either in terms of any other type of adverse event, respiratory assistance needs ( 11 versus 10 patients, respectively; $\mathrm{p}=0.89$ ) nor in terms of the length of stay ( $13.3 \pm 11.3$ versus $12.5 \pm 12.4$ days, respectively; $\mathrm{p}=0.7$ ) in the standard and automated groups.

\section{Discussion}

The $\mathrm{FreeO}_{2}$ PostOp trial was a pragmatic international multicentre randomised clinical trial designed to test the hypothesis that automated closed-loop $\mathrm{O}_{2}$ administration is superior to standard manual $\mathrm{O}_{2}$ administration in patients recovering from high-risk abdominal or thoracic surgery. To the best of our knowledge, this is the first study to investigate the usefulness of automated $\mathrm{O}_{2}$ administration in such an indication. Automated $\mathrm{O}_{2}$ administration was demonstrated to increase time within the oxygenation range following high-risk surgery, as compared to standard $\mathrm{O}_{2}$ administration. In addition, there was a significant decrease in the occurrence of hypoxaemia and hyperoxaemia for patients assigned to the automated group.

\begin{tabular}{|c|c|c|c|}
\hline & Total & Standard & Automated \\
\hline Subjects & 199 & 103 & 96 \\
\hline Age years & $62.7 \pm 13.0$ & $63.1 \pm 13.5$ & $62.2 \pm 12.6$ \\
\hline Male & 110 (55.3) & 57 (55.3) & $53(55.2)$ \\
\hline COPD & 17 (8.5) & $10(9.7)$ & 7 (7.3) \\
\hline \multicolumn{4}{|l|}{ Surgery characteristics } \\
\hline Abdominal & $141(71.2)$ & 74 (71.8) & $67(70.5)$ \\
\hline Thoracic & $57(28.8)$ & 29 [28.2] & 28 (29.5) \\
\hline \multicolumn{4}{|l|}{ ARISCAT risk class } \\
\hline Moderate risk (26-44) & $150(75.4)$ & 78 (75.7) & $72(75.0)$ \\
\hline High risk ( $\geqslant 45)$ & $49(24.6)$ & 25 (24.3) & $24(25.0)$ \\
\hline \multicolumn{4}{|l|}{ Comorbidities } \\
\hline Alcohol abuse & $26(14.9)$ & $11(12.1)$ & $15(17.9)$ \\
\hline Diabetes & $26(14.8)$ & $13(14.1)$ & $13(15.5)$ \\
\hline \multicolumn{4}{|l|}{ Cardiovascular } \\
\hline Arterial hypertension & $67(37.9)$ & $32(34.8)$ & $35(41.2)$ \\
\hline Coronaropathy & $17(9.7)$ & $9(9.8)$ & $8(9.5)$ \\
\hline Cardiac insufficiency & $2(1.1)$ & $0(0)$ & $2(2.4)$ \\
\hline \multicolumn{4}{|l|}{ Respiratory } \\
\hline Allergy & $18(10.3)$ & $15(16.5)$ & $3(3.6)$ \\
\hline Smokers & $101(57.7)$ & $48(52.7)$ & $53(63.1)$ \\
\hline Asthma & $7(4.0)$ & $5(5.5)$ & $2[2.4]$ \\
\hline LTOT & $1(0.6)$ & $1(1.1)$ & $0(0)$ \\
\hline Home ventilation & $0(0)$ & $0(0)$ & $0(0)$ \\
\hline Renal insufficiency & $14(8.0)$ & $8(8.7)$ & $6(7.1)$ \\
\hline \multicolumn{4}{|l|}{ Physiological parameters } \\
\hline Pain control ${ }^{\#}$ & $2.8 \pm 3.1$ & $3.0 \pm 3.1$ & $2.6 \pm 3.2$ \\
\hline Respiratory rate breaths $\cdot \min ^{-1}$ & $16 \pm 5$ & $16 \pm 5$ & $16 \pm 4$ \\
\hline $\mathrm{S}_{\mathrm{pO}_{2}} \%$ & $97.6 \pm 2.5$ & $98.0 \pm 2.5$ & $97.2 \pm 2.3$ \\
\hline Respiratory comfort ${ }^{\#}$ & $9.1 \pm 1.5$ & $9.0 \pm 1.5$ & $9.2 \pm 1.5$ \\
\hline Heart rate beats $\min ^{-1}$ & $82 \pm 16$ & $81 \pm 16$ & $83 \pm 15$ \\
\hline Systolic arterial pressure $\mathrm{mmHg}$ & $124 \pm 23$ & $123 \pm 23$ & $126 \pm 22$ \\
\hline Mean arterial pressure $\mathrm{mmHg}$ & $85 \pm 15$ & $84 \pm 15$ & $87 \pm 15$ \\
\hline
\end{tabular}

Data are presented as $n$, mean \pm SD or $n(\%)$. There were no significant differences between the study groups in any of the characteristics listed. Patients who were assigned to receive automated oxygen $\left(\mathrm{O}_{2}\right)$ administration were connected to the $\mathrm{FreeO}_{2}$ system, set on the automated mode. Patients assigned to standard $\mathrm{O}_{2}$ administration were also connected to the $\mathrm{Free}_{2}$ system for monitoring purposes, but the system was set solely to the recording mode. $\mathrm{O}_{2}$ flow was the value measured immediately after randomisation; the flow was significantly lower in the automated group while $\mathrm{FreeO}_{2}$ takes only several seconds to adjust to the real patients' needs, thus rapidly weaning patients who no longer require $\mathrm{O}_{2}$. Treatment limitation and do-not-intubate order was systematically assessed on admission by either the emergency physician or the intensivist, according to patient's health status. ARISCAT: Assess Respiratory Risk in Surgical Patients in Catalonia; a score that predicts the risk of pulmonary complications after surgery, including respiratory failure. To be randomised, patients had to have a predicted ARISCAT score $\geqslant 26$ prior to surgery; LTOT: long-term oxygen therapy; $S_{\mathrm{pO}_{2}}$ : oxygen saturation measured by pulse oximetry. ": assessed using hetero-evaluation by the anaesthesiology nurse in the recovery room, using analogic numerical scale (from 1 to $10 ; 1$ being the worst and 10 the best value). 
Post-operative short-term (recovery room; $3 \mathrm{~h}$ )

\begin{tabular}{lll}
\hline Automated Standard & $\begin{array}{c}\text { Adjusted } \\
\text { difference } \\
(95 \% \mathrm{CI})\end{array}$
\end{tabular}

Post-operative long-term (surgical ward; up to 3 days)

\begin{tabular}{lll}
\hline Automated Standard & $\begin{array}{c}\text { Adjusted } \\
\text { difference } \\
(95 \% \mathrm{CI})\end{array}$
\end{tabular}

\begin{tabular}{|c|c|c|c|c|c|c|c|c|}
\hline $\mathrm{S}_{\mathrm{po}_{2}} \%$ & $94.7 \pm 4.1$ & $96.2 \pm 3.1$ & $-1.6(-2.6--0.5)$ & 0.004 & $95.2 \pm 1.3$ & $94.6 \pm 2.7$ & $0.6(0-1.2)$ & 0.09 \\
\hline Time within range ${ }^{\#}$ min & $156 \pm 32$ & $71 \pm 63$ & $85(69-100)$ & $<0.0001$ & $2694 \pm 1134$ & $\begin{array}{r}1969 \\
\pm 1031\end{array}$ & $714(401-1027)$ & $<0.0001$ \\
\hline Time within range ${ }^{\#} \%$ & $91.4 \pm 13.7$ & $\begin{array}{r}40.2 \\
\pm 35.1\end{array}$ & $51.0(42.8-59.2)$ & & $94.0 \pm 11.3$ & $62.1 \pm 23.3$ & $31.9(26.3-37.4)$ & \\
\hline Hypoxaemia ${ }^{\eta} \min$ & $2.8 \pm 4.9$ & $7.8 \pm 18.4$ & $-5.0(-9.2--0.8)$ & 0.02 & $31 \pm 57$ & $373 \pm 591$ & $\begin{array}{c}-342(-471- \\
-213)\end{array}$ & $<0.0001$ \\
\hline Severe hypoxaemia $^{+} \min$ & $0.1 \pm 0.3$ & $0.3 \pm 1.0$ & $-0.1(-0.4-0.1)$ & 0.23 & $2 \pm 3$ & $10 \pm 27$ & $-8(-14--2)$ & 0.007 \\
\hline Hyperoxaemia ${ }^{\S} \min$ & $11.9 \pm 22.6$ & $\begin{array}{c}97.3 \\
\pm 68.4\end{array}$ & $\begin{array}{c}-85.2(-100.9- \\
-69.5)\end{array}$ & $<0.0001$ & $148 \pm 401$ & $800 \pm 716$ & $-643(-818--469)$ & $<0.0001$ \\
\hline $\begin{array}{l}\text { Severe hyperoxaemia } f \\
\text { min }\end{array}$ & $0.9 \pm 3.2$ & $\begin{array}{r}45.2 \\
\pm 57.8\end{array}$ & $\begin{array}{c}-44.6(-57.2- \\
-31.9)\end{array}$ & $<0.0001$ & $4 \pm 7$ & $174 \pm 274$ & $-172(-232--113)$ & $<0.0001$ \\
\hline $\mathrm{S}_{\mathrm{po}_{2}} 100 \% \min$ & $0.3 \pm 1.5$ & $\begin{array}{r}19.3 \\
\pm 36.5\end{array}$ & $\begin{array}{c}-19.2(-27.2- \\
-11.1)\end{array}$ & $<0.0001$ & $1 \pm 2$ & $46 \pm 82$ & $-45(-63--28)$ & $<0.0001$ \\
\hline
\end{tabular}

The oxygen $\left(\mathrm{O}_{2}\right)$ flow that is depicted within the table is the mean value during the recording windows. Time within range is significantly increased for patients assigned to the automated group, either during the short-term and long-term post-operative periods ( $p<0.0001$ ). This result is explained by a huge difference in terms of $\mathrm{O}_{2}$ flow adjustments in response to the patients' needs, thus inducing a reduction of time with hypoxaemia and hyperoxaemia. Severe hypoxaemia is decreased during the 3-day recording in patients assigned to the automated group, while $\mathrm{O}_{2}$ weaning (defined as a mean $\mathrm{O}_{2}$ flow $\leqslant 0.3 \mathrm{~L} \cdot \mathrm{min}^{-1}$ during the last $10 \mathrm{~min}$ of the short-term post-operative phase or during the last $1 \mathrm{~h}$ of the long-term post-operative phase) is higher in the standard group, but time without $\mathrm{O}_{2}$ is higher in the automated group. Such results may suggest that continuous monitoring and $\mathrm{O}_{2}$ adjustment to the needs of such patients might be warranted for up to 3 days following high-risk surgery. Bold type represents statistical significance $(p \leqslant 0.05)$. $S_{\mathrm{pO}_{2}}$ : oxygen saturation measured by pulse oximetry $\#$ : Main study objective;

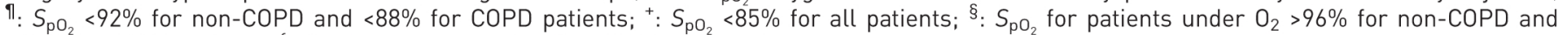
$>92 \%$ for COPD patients; ${ }^{f}: \mathrm{S}_{\mathrm{pO}_{2}}$ for patients under $\mathrm{O}_{2}>98 \%$ for all patients.

TABLE 3 Secondary outcomes

\begin{tabular}{|c|c|c|c|c|}
\hline & Automated & Standard & $\begin{array}{c}\text { Adjusted difference/ } \\
\text { OR }(95 \% \mathrm{CI})\end{array}$ & p-value \\
\hline \multicolumn{5}{|l|}{ Recovery room } \\
\hline Treatment duration min & $183 \pm 14$ & $180 \pm 13$ & $-2(-6-2)$ & 0.26 \\
\hline $\mathrm{O}_{2}$ flow $\mathrm{L} \cdot \mathrm{min}^{-1}$ & $0.9 \pm 1.4$ & $2.8 \pm 1.4$ & $-1.9(-2.3--1.5)$ & $<0.0001$ \\
\hline $\mathrm{O}_{2}$ flow variations ${ }^{\#} \mathrm{n}$ & $10290 \pm 1352$ & $2 \pm 1$ & & $<0.0001$ \\
\hline Time without $\mathrm{O}_{2} \mathrm{~min}$ & $87.6 \pm 59.5$ & $17.4 \pm 41.9$ & $70.3(55.2-85.3)$ & $<0.0001$ \\
\hline $\mathrm{O}_{2}$ weaning ${ }^{9}$ & 50 (61.7) & $14(16.3)$ & $8.9(4.2-18.8)$ & $<0.0001$ \\
\hline \multicolumn{5}{|l|}{ Surgical ward } \\
\hline Treatment duration min & $3146 \pm 1225$ & $3506 \pm 982$ & $-357(-682--33)$ & 0.03 \\
\hline $\mathrm{O}_{2}$ flow $\mathrm{L} \cdot \mathrm{min}^{-1}$ & $1.0 \pm 1.3$ & $1.2 \pm 1.0$ & $-0.2(-0.6-0.1)$ & 0.15 \\
\hline $\mathrm{O}_{2}$ flow variations ${ }^{\#} \mathrm{n}$ & $174030 \pm 69484$ & $6 \pm 3$ & & $<0.0001$ \\
\hline Time without $\mathrm{O}_{2} \mathrm{~min}$ & $1371 \pm 1104$ & $1261 \pm 1170$ & $109(-232-450)$ & 0.53 \\
\hline $\mathrm{O}_{2}$ weaning 9 & 35 (42.2) & $50(58.1)$ & $0.5(0.3-1.0)$ & 0.04 \\
\hline \multicolumn{5}{|l|}{ Day 28} \\
\hline Mortality & $3(3.2)$ & $1(1.0)$ & $3.3(0.3-32.0)$ & 0.31 \\
\hline Respiratory assistance & $10(10.4)$ & $11(10.7)$ & $1.0(0.4-2.4)$ & 0.94 \\
\hline Hospital length of stay days & $12.5 \pm 12.4$ & $13.3 \pm 11.3$ & $-0.6(-3.9-2.6)$ & 0.7 \\
\hline \multicolumn{5}{|c|}{$\begin{array}{l}\text { Data are presented as mean } \pm \text { SD or } \mathrm{n}(\%) \text {, unless otherwise stated. Treatment duration was similar in both } \\
\text { groups in the recovery room, but higher in the standard group within the surgical ward. The oxygen }\left(\mathrm{O}_{2}\right) \\
\text { flow that is depicted within the table is the mean value during the recording windows; no differences were } \\
\text { observed in between groups. } \mathrm{O}_{2} \text { weaning is higher in the standard group, but time without } \mathrm{O}_{2} \text { is higher in } \\
\text { the automated group. }{ }^{\#} \text { : the absolute number of changes in the } \mathrm{O}_{2} \text { flow during the follow-up period; }{ }^{n}: \text { a } \\
\text { mean } \mathrm{O}_{2} \text { flow } \leqslant 0.3 \mathrm{~L} \cdot \mathrm{min}^{-1} \text { during the last } 10 \text { min of the short-term post-operative phase (recovery room) } \\
\text { or during the last } 1 \mathrm{~h} \text { of the long-term post-operative phase. Bold type represents statistical significance } \\
(p \leqslant 0.05) \text {. }\end{array}$} \\
\hline
\end{tabular}



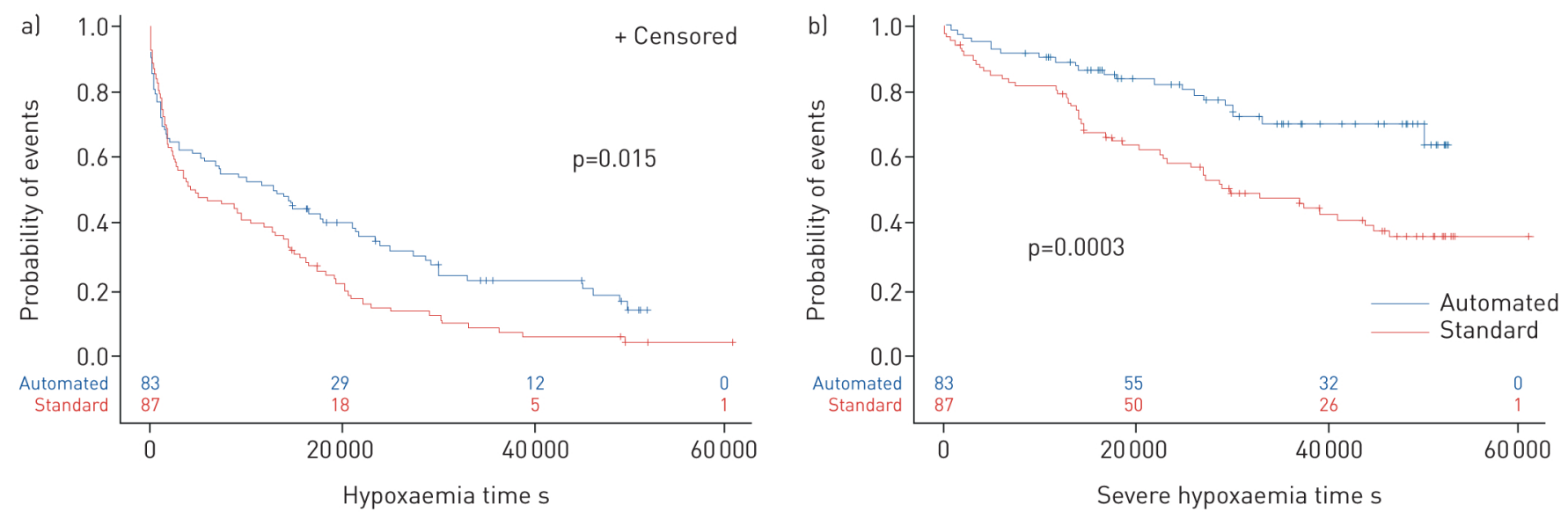

FIGURE 2 Kaplan-Meier estimate of hypoxaemia occurrence in both groups. Events are defined as the first occurrence of either a) a clinically significant hypoxaemia loxygen $\left(\mathrm{O}_{2}\right)$ saturation measured by pulse oximetry $\left(\mathrm{S}_{\mathrm{pO}_{2}}\right)<92 \%$ for non-COPD patients, <88\% for COPD patients) or b) a severe hypoxaemia $\left(S_{\mathrm{pO}_{2}} \leqslant 85 \%\right.$ for all patients), for more than a consecutive 30-s period. The evaluation was performed over a 3-day period. Censored patients are those for whom $\mathrm{O}_{2}$ was removed during monitoring, before the end of the maximal 3 days of follow-up, because of either weaning and/or clinical improvement. Analysis was performed over $S_{\mathrm{pO}_{2}}$ recordings of all patients, providing one value each second. Both probability conditions were significantly reduced in the automated as compared to the standard group ( $p=0.015$ for hypoxaemia, and $p=0.0003$ for severe hypoxaemial. Numbers in blue depict the remaining patients without event occurrence in the automated group; numbers in red depict the remaining patients in the standard group.

\section{Impact on hypoxaemia}

There is a good level of evidence and good acceptance by the medical community that hypoxaemia is harmful [26], especially in adult patients with myocardial ischaemia [27] or neurotrauma [28]. In addition, the data suggest that even short periods of hypoxaemia may promote significant negative haemodynamic effects [29]. In an animal model, right ventricular dilation was observed with only $2 \mathrm{~h}$ of daily hypoxaemia [30].

In post-operative patients, pulmonary function is altered by general anaesthesia and surgery. Post-operative respiratory complications following surgery are the second most frequent complications [19] and considered as a major cause of morbidity and mortality $[17,24,25]$. In this condition, the occurrence of oxygen desaturation related to periodic apnoea and hypoventilation has been recognised for a long time [31, 32], with potentially severe consequences such as myocardial ischaemia [33]. The incidence of hypoxaemia is high in the recovery room (10-50\%) [20], and up to $50 \%$ of post-operative patients will demonstrate episodic hypoxaemia in the absence of oxygen therapy $[34,35]$. It has also been shown that desaturation episodes are more frequent during the first night following surgery, but may also be worsened 3-5 days post-operatively, especially in patients with obstructive sleep apnoea [36-38]. In a study performed on 833 patients with continuous oximetry monitoring following non-cardiac surgery, hypoxaemia was found to be common and prolonged, even in patients without specific risk factors [22]. The authors pointed out the fact that conventional point-check oximetry within surgical wards may underestimate hypoxaemia incidence and severity, and postulated that point-check monitoring on aroused patients may induce $S_{\mathrm{pO}_{2}}$ measurements increases that might be unsustained once the stimulus is removed, thus masking oxygen desaturations.

The pathophysiology of these oxygen desaturations is rather complex, but may be due to the patient's condition itself (advanced age, COPD, diabetes, obesity) or modifications in respiratory mechanics (reduction of functional residual capacity, atelectasis, thoracoabdominal compliance decrease), but also to the use of pharmaceutical agents that are given during surgery (anaesthetics and neuromuscular blocking drugs), or those that are given to relieve post-operative pain (opioids and sedatives) [32]. In our study, hypoxaemia periods were shorter in the automated group (adjusted difference $-95 \mathrm{~min} ; \mathrm{p}<0.0001$ ), less frequent, but the incidence was also significantly lower, as 25 and 17 patients experienced hypoxaemia for $>10 \%$ and $>20 \%$ of the time, respectively, in the standard group, as compared to none within the automated group during the 3 -day period $(\mathrm{p}<0.001)$. It is somewhat interesting that hypoxaemia episodes within the standard group occurred despite frequent hyperoxaemia within the first 3-h period (supplementary figure S2). These hypoxaemia episodes remained frequent during the 3 days following surgery (supplementary figure S3), probably because of underlying patient pathologies and the use of opioid analgesics.

\section{Impact on hyperoxaemia}

It has been demonstrated in two randomised trials by our team $[10,15]$ that time with hyperoxaemia could be reduced with automated closed-loop oxygen administration, and we initially hypothesised that this problem would also be reduced after thoracic or abdominal surgeries. 
Within our study, supplemental oxygen was overwhelmingly used during the immediate post-operative phase, thus promoting excessive hyperoxaemia (adjusted difference of time within range $+51 \%, 42.8-$ $59.2 \% ; \mathrm{p}<0.0001)$ without suppression of hypoxaemia periods in the standard group. Such overoxygenation is very frequent in most patients within the recovery room, even in COPD patients, and oxygen flow adjustments in response to $\mathrm{S}_{\mathrm{pO}_{2}}$ values are quite few (supplementary figure S3).

Few studies have promoted the utilisation of hyperoxaemia for colorectal surgery, in order to reduce wound infection [39], but this is not recommended in routine given controversial data [40-42]. In a post hoc analysis of the PROXI trial, the authors pointed out the potential risks of acute coronary syndromes associated with perioperative hyperoxaemia [43]. The pathophysiological risks associated with hyperoxaemia have been recognised for a long time, especially in COPD patients [4]. The first recommendation to adjust oxygen flow rates in order to reduce the risks of hyperoxaemia was published in the early 1960s [44], and several more recent guidelines have reiterated similar recommendations $[1,2]$. The recent demonstration in a large randomised controlled trial of increased mortality in ICU patients assigned to standard oxygen therapy practice as compared to a more conservative one (absolute risk reduction $0.086,95 \%$ CI $0.017-0.150 ; \mathrm{p}=0.01$ ) has revived the debate about the potential harm of excessive oxygen therapy in an unselected patient population [45]. Adverse effects of hyperoxaemia could be mediated through higher oxidative stress, but also increased coronary $[6,7]$ and cerebral artery resistance [8], which are all associated with a potential clinical impact [46, 47]. Another side-effect of oxygen is caused by gas absorption, which is a known mechanism of atelectasis formation. While several other observational studies have supported similar benefits when avoiding hyperoxaemia and liberal oxygen use $[48,49]$, the recent ICU-ROX and $\mathrm{LOCO}_{2}$ trials $[50,51]$ depicted conflicting results without clear evidence of any benefit of avoiding hyperoxaemia, but rather potential harm related to a low $S_{\mathrm{pO}_{2}}$ value.

Even if debated, the potential physiological adverse events $[6-8,46,52]$ related to hyperoxaemia clearly mandates attention in avoiding unnecessary oxygen administration, i.e. not administering oxygen when the $\mathrm{S}_{\mathrm{pO}_{2}}$ is $\geqslant 96 \%$, and not starting oxygen when the $S_{\mathrm{pO}_{2}}$ is $92 \%$ or $93 \%[53,54]$. The DETO2X-AMI randomised trial in patients with acute myocardial infarction provides definitive evidence for a lack of benefit for supplemental oxygen therapy in patients with acute myocardial infarction who have a normal $S_{\mathrm{pO}_{2}}$ value [55].

\section{Automated weaning of oxygen}

Oxygen supplementation is almost invariably applied after elective extubation to correct oxygenation impairment and to decrease the occurrence of post-operative hypoxaemia, even though it is well known that it will not have any effect on the overall number of central or obstructive apnoea events nor atelectasis [31]. Not all patients will benefit from systematic oxygen administration following endotracheal extubation [56], but those with a high-risk profile probably will, especially following long-duration and major thoracic or abdominal surgery, such as those patients recruited within our study [57-59]. Moreover, standard continuous oxygen administration reduces, but does not abolish the occurrence of desaturation [34], given the fact that solely $S_{\mathrm{pO}_{2}}$ point checks are made to adjust oxygen flow to actual patient needs, thus missing the occurrence of transient hypoxaemia.

It has also been demonstrated that the duration of oxygen therapy is an independent risk factor for developing post-operative respiratory complications. Patients who require oxygen for $\geqslant 75 \%$ of recovery room time (or $>90 \mathrm{~min}$ ) appear to be at a greater risk of developing respiratory complications [60]. This fact may suggest that some patients are not adequately screened for risk factors such as obstructive sleep apnoea by standard pre-anaesthesia testing, and that a device dedicated to continuous monitoring of oxygen administration (alarms on oxygen administration duration and flow variations) may help to detect such high-risk patients. The increased resource utilisation in patients with longer oxygen therapy requirements in the recovery room likely reflects the increase in the occurrence of pulmonary respiratory complications requiring invasive and noninvasive ventilatory support, especially on the day of surgery. Unfortunately, our study was not powered to enable such outcome impact evaluation. In a study by L'HeR et al. [10] performed within the emergency department environment, it was shown that the partial or complete oxygen weaning was significantly increased with automated oxygen titration in comparison with standard oxygen administration.

In the specific setting of post-operative patients, a reduction in weaning time may improve the efficiency of the turnover of the patients in the recovery room. However, it must not be forgotten that patient monitoring and treatment with automated closed-loop administration during the entire 3-day period following these high-risk surgeries may also be beneficial, while it may significantly decrease the occurrence of significant hypoxaemia. 
Several systems have been developed to titrate oxygen flow rate in neonates and in adult patients $[10,14]$. In a previous randomised controlled trial on adult patients admitted to the emergency department for acute hypoxaemic respiratory failure, the use of automated oxygen administration was found superior to manual oxygen administration to improve the time spent within oxygenation targets, with a between-group difference of $29 \%$ [10], as already observed in other studies on automated oxygen administration [61, 62]. In a study by L'Her et al. [10] performed in the stabilisation units of emergency departments, patients experienced less time with hypoxaemia and hyperoxaemia in the $\mathrm{FreeO}_{2}$ group. When receiving automated oxygen, partial or complete oxygen weaning was more frequent during initial care, as compared to standard manual oxygen administration. Considering safety issues, the automated system continuously adjusts the oxygen flow in response to patient needs, and these $\mathrm{O}_{2}$ flow variations are continuously visualised on the screen. While the closed-loop system requires continuous $S_{\mathrm{pO}_{2}}$ monitoring to adjust the flow, this means that, on a routine basis, the patient is monitored for $\mathrm{S}_{\mathrm{pO}_{2}}, \mathrm{O}_{2}$ flow, respiratory rate and heart rate [63]; each of these parameters are monitored using adjustable alarm levels to standards or individualised goals, and deviation from the range is either visualised by lights and/or sounds. Furthermore, all trends for these parameters can be visualised and downloaded.

\section{Study limitations}

The first limitation of the study is that investigators were aware of the inclusion group, as blinding was difficult for technical reasons. As stated within the methods section, all participating units were encouraged to use the same oxygenation target in both groups, and staffing levels were equivalent. Within the recovery room, $\mathrm{O}_{2}$ flow was regulated by anaesthesiology wards, and within the surgical ward it was regulated by general nurses, according to the physician's prescription. However, because patients in the standard group were also monitored with the $\mathrm{FreeO}_{2}$ device in recording mode, we cannot ensure that patients were not monitored more closely than in routine, especially within surgical wards. Such bias was the only technical way to ensure continuous oximetry recordings with the same sampling rate $(1 \mathrm{~Hz})$. However, such bias may only have decreased the potential to identify a difference between groups (i.e. to decrease the benefits of automated closed-loop oxygen administration), while a significant difference was demonstrated. Second, the assessment of oxygenation status could be considered more precise by analysing blood gas samples rather than $S_{\mathrm{pO}_{2}}$. However, this would not enable continuous oxygenation monitoring for up to 3 days. Moreover, only continuous of nonaveraged $S_{\mathrm{pO}}$, values enable precise and rapid adjustments of the oxygen flow in response to exact patient needs. Of note, all patients in both groups were continuously monitored using the same oximeter, which may represent a strength of this study; therefore, the $\mathrm{FreeO}_{2}$ PostOp study represents the largest prospective study comparing two oxygenation strategies over such a period in the post-operative setting. Third, our study was not powered for major respiratory complications nor with the modification of other adverse outcomes. However, while hypoxaemia is widely thought to be harmful, such differences in terms of time within the oxygenation range might be considered as beneficial.

In conclusion, hypoxaemia and hyperoxaemia were common and prolonged in patients recovering from high-risk surgery and assigned to standard oxygen administration. Automated closed-loop oxygen administration promoted a greater proportion of time within the $S_{\mathrm{pO}_{2}}$ target range, thus avoiding prolonged periods of hypoxaemia and hyperoxaemia.

Author contributors: E. L'Her and F. Lellouche designed this study, drafted the manuscript of the protocol and critically revised the manuscript. E. L'Her, S. Jaber, D. Verzilli, C. Jacob, B. Huiban, E. Futier, T. Kerforne, V. Pateau, P-A. Bouchard, M. Consigny and F. Lellouche participated in the conduct of the study. M. Consigny and E. Nowak participated in the protocol methodological assessment and statistical plan. All authors read and approved the final manuscript.

Ethics approval: The institutional review board of the University Hospital of Brest (France) approved the trial for all French centres (IDRCB RB14-060). The institutional review board from the Québec Heart and Lung Institute (Canada) approved the trial for their own centre. Any protocol modification will be submitted for review and approval by the ethics committee. The FreeO $\mathrm{O}_{2}$ Post-Op study is conducted in accordance with the declaration of Helsinki and was registered on September 11, 2015 at http://www.clinicaltrials.gov with trial identification number NCT02546830. First patient inclusion was performed on January 14, 2016.

Conflict of interest: E. L'Her reports other from Oxynov, during the conduct of the study; personal fees from Smiths Medical, personal fees and other from GE Healthcare, grants and personal fees from Sedana Medical, outside the submitted work. In addition, E. L'Her has a patent Method and device for delivering oxygen licensed to Oxynov. S. Jaber reports personal fees from Drager, Fisher-Paykel, Baxter, Fresenius-Xenios and Medtronic, during the conduct of the study. D. Verzilli has nothing to disclose. C. Jacob has nothing to disclose. B. Huiban has nothing to disclose. E. Futier reports consulting fees from Drager Medical, GE Healthcare, Orion Pharma and Edwards Lifesciences, lecture fees from Fresenius Kabi and Getinge, and non-financial support from Fisher and Paykel Healthcare, during the conduct of the study. T. Kerforne has nothing to disclose. V. Pateau reports other from OxyNov, during the conduct of 
the study. P-A. Bouchard has nothing to disclose. M. Consigny has nothing to disclose. F. Lellouche reports other from Oxynov, during the conduct of the study.

Support statement: This trial was funded by the French Ministry of Health obtained in 2014 from a regional hospital clinical research programme (Programme Hospitalier de Recherche Clinique Interrégional HUGO 2012-199). The sponsors had no role in the study design and conduct; the collection, management, analysis and interpretation of the data; or the preparation and approval of the manuscript. Funding information for this article has been deposited with the Crossref Funder Registry.

\section{References}

1 O'Driscoll BR, Howard LS, Earis J, et al. BTS guidelines for oxygen use in adults in healthcare and emergency settings. Thorax 2017; 72: Suppl. 1, iil-ii90.

2 GOLD Scientific Committee. Global Strategy for the Diagnosis, Management, and Prevention of Chronic Obstructive Pulmonary Disease 2016. Available from: http://goldcopd.org/ Date last accessed: March 30, 2018.

3 Abdo WF, Heunks LMA. Oxygen-induced hypercapnia in COPD: myths and facts. Crit Care 2012; 16: 323.

4 Aubier M, Murciano D, Milic-Emili J, et al. Effects of the administration of $\mathrm{O} 2$ on ventilation and blood gases in patients with chronic obstructive pulmonary disease during acute respiratory failure. Am Rev Respir Dis 1980; 122: $747-754$.

5 Austin MA, Wills KE, Blizzard L, et al. Effect of high flow oxygen on mortality in chronic obstructive pulmonary disease patients in prehospital setting: randomized controlled trial. BMJ 2010; 341: c5642.

6 Wijesinghe $\mathrm{M}$, Perrin $\mathrm{K}$, Ranchord $\mathrm{A}$, et al. Routine use of oxygen in the treatment of myocardial infarction: systematic review. Heart 2009; 95: 198-202.

7 Farquhar H, Weatherall M, Wijesinghe $\mathrm{M}$, et al. Systematic review of studies of the effect of hyperoxia on coronary blood flow. Am Heart J 2009; 158: 371-377.

8 Floyd TF, Clark JM, Gelfand R, et al. Independent cerebral vasoconstrictive effects of hyperoxia and accompanying arterial hypocapnia at 1 ATA. J Appl Physiol 2003; 95: 2453-2461.

9 Mok W, Wang W, Cooper S, et al. Attitudes towards vital signs monitoring in the detection of clinical deterioration: scale development and survey ward nurses. Int J Qual Health Care 2015; 27: 207-213.

10 L'Her E, Dias P, Gouillou M, et al. Automatic versus manual oxygen administration in the emergency department. Eur Respir J 2017; 50: 1602552.

11 Claure N, Gerhardt T, Everett R, et al. Closed-loop controlled inspired oxygen concentration for mechanically ventilated very low birth weight infants with frequent episodes of hypoxemia. Pediatrics 2001; 107: 1120-1124.

12 Claure N, Bancalari E, D’Ugard C, et al. Multicenter crossover study of automated controlled of inspired oxygen in ventilated preterm infants. Pediatrics 2011; 127: e76-e83.

13 Hallenberger A, Poets CF, Horn W, et al. Closed-loop automatic oxygen control (CLAC) in preterm infants: a randomized controlled trial. Pediatrics 2014; 133: e379-e385.

14 Lellouche F, L'Her E. Automated oxygen flow titration to maintain constant oxygenation. Respir Care 2012; 57: $1254-1262$.

15 Lellouche F, Bouchard PA, Roberge M, et al. Automated oxygen titration and weaning with FreeO2 in patients with acute exacerbation of COPD: a pilot randomized trial. Int J Chron Obstruct Pulmon Dis 2016; 11: 1983-1990.

16 Weiser TG, Regenbogen SE, Thompson $\mathrm{KD}$, et al. An estimation of the global volume of surgery: a modelling strategy based on available data. Lancet 2008; 372: 139-144.

17 Khuri SF, Henderson WG, DePalma RG, et al. Determinants of long-term survival after major surgery and the adverse effect of postoperative complications. Ann Surg 2005; 242: 326-341.

18 Shander A, Fleisher LA, Barie PS, et al. Clinical and economic burden of postoperative pulmonary complications: patient safety summit on definition, risk-reducing interventions, and preventive strategies. Crit Care Med 2011; 39: $2163-2172$.

19 Arozullah AM, Daley J, Henderson WG, et al. Multifactorial risk index for predicting postoperative respiratory failure in men after major noncardiac surgery. The National Veterans Administration Surgical Quality Improvement Program. Ann Surg 2000; 232: 242-253.

20 Lawrence VA, Dhanda R, Hilsenbeck SG, et al. Risk of pulmonary complications after elective abdominal surgery. Chest 1996; 110: 744-750.

21 Lindberg P, Gunnarsson L, Tokics L, et al. Atelectasis and lung function in the postoperative period. Acta Anaesthesiol Scand 1992; 36: 546-553.

22 Sun Z, Sessler DI, Dalton JE, et al. Postoperative hypoxemia is common and persistent: a prospective blinded observational study. Anesth Analg 2015; 121: 709-715.

23 Gerber D, Guensch DP, Theiler L, et al. When less is more: why extubation with less than routine $100 \%$ oxygen may be a reasonable strategy. Anesth Analg 2019; 129: 1433-1435.

24 Mazo V, Sabaté S, Canet J, et al. Prospective external validation of a predictive score for postoperative pulmonary complications. Anesthesiology 2014; 121: 219-231.

25 L'Her E, Jaber S, Verzilli D, et al. Automated oxygen administration versus conventional oxygen therapy after major abdominal or thoracic surgery: study protocol for an international multicentre randomised controlled study. BMJ Open 2019; 9: e023833.

26 Bateman NT, Leach RM. ABC of oxygen. Acute oxygen therapy. BMJ 1998; 317: 798-801.

27 Galatius-Jensen S, Hansen J, Rasmussen V, et al. Nocturnal hypoxaemia after myocardial infarction: association with nocturnal myocardial ischaemia and arrythmias. Br Heart J 1994; 72: 23-30.

28 DeWitt DS, Prough DS. Blast-induced brain injury and posttraumatic hypotension and hypoxemia. J Neurotrauma 2009; 26: 877-887.

29 Selinger SR, Kennedy TP, Buescher P, et al. Effects of removing oxygen from patients with chronic obstructive disease. Am Rev Respir Dis 1987; 136: 85-91.

30 Nattie EE, Doble EA. Threshold of intermittent hypoxia-induced right ventricular hypertrophy in the rat. Respir Physiol 1984; 56: 253-259. 
31 Jones JG, Jordan C, Scudder C, et al. Episodic postoperative oxygen desaturation: the value of added oxygen. $J R$ Soc Med 1985; 78: 1019-1022.

32 Catley DM, Thornton C, Jordan C, et al. Pronounced, episodic oxygen desaturation in the postoperative period: its association with ventilatory pattern and analgesic regimen. Anesthesiology 1985; 63: 20-28.

33 Gögenur I, Rosenberg-Adamsen S, Lie C, et al. Relationship between nocturnal hypoxaemia, tachycardia and myocardial ischaemia after major abdominal surgery. Br J Anaesth 2004; 93: 333-338.

34 Canet J, Ricos M, Vidal F. Early postoperative arterial oxygen desaturation. Determining factors and response to oxygen therapy. Anesth Analg 1989; 69: 207-212.

35 Ramanchandran SK, Nafiu OO, Ghaferi A, et al. Independent predictors and outcomes of unanticipated early postoperative tracheal intubation after nonemergent, noncardiac surgery. Anesthesiology 2011; 115: 44-53.

36 Gögenur I, Wildschiotz G, Rosenberg J. Circadian distribution of sleep phases after major abdominal surgery. $\mathrm{Br} J$ Anaesth 2008; 100: 45-49.

37 Chung F, Liao P, Elsaid H, et al. Factors associated with postoperative exacerbation of sleep-disordered breathing. Anesthesiology 2014; 120: 299-311.

38 Rosenberg J, Wildschiødtz G, Pedersen MH, et al. Late postoperative nocturnal episodic hypoxaemia and associated sleep pattern. Br J Anaesth 1994; 72: 145-150.

39 Greif R, Akça O, Horn EP, et al. Supplemental perioperative oxygen to reduce the incidence of surgical-wound infection. N Engl J Med 2000; 342: 161-167.

40 Meyhoff CS, Wetterslev J, Jorgensen LN, et al. Effect of high perioperative oxygen fraction on surgical site infection and pulmonary complications after abdominal surgery: the PROXI randomized clinical trial. JAMA 2009; 302: $1543-1550$.

41 Togioka B, Galvagno S, Sumida S, et al. The role of perioperative high inspired oxygen therapy in reducing surgical site infection: a meta-analysis. Anesth Analg 2012; 114: 334-342.

42 Wetterslev J, Meyhoff CS, Jørgensen LN, et al. The effects of high perioperative inspiratory oxygen fraction for adult surgical patients. Cochrane Database Syst Rev 2015; 2015: CD008884.

43 Fonnes S, Gögenur I, Sondergaard ES, et al. Perioperative hyperoxia - long-term impact on cardiovascular complications after abdominal surgery, a post hoc analysis of the PROXI trial. Int J Cardiol 2016; 215: 238-243.

44 Campbell EJ. The J. Burns Amberson Lecture. The management of acute respiratory failure in chronic bronchitis and emphysema. Am Rev Respir Dis 1967; 96: 626-639.

45 Girardis M, Busani S, Damiani E, et al. Effect of conservative vs conventional oxygen therapy on mortality among patients in an intensive care unit: the Oxygen-ICU randomized clinical trial. JAMA 2016; 316: 1583-1589.

46 Stub D, Smith K, Bernard S, et al. Air versus oxygen in ST-segment-elevation myocardial infarction. Circulation 2015; 131: 2143-2150.

47 Damiani E, Adrario E, Girardis M, et al. Arterial hyperoxia and mortality in critically ill patients: a systematic review and meta-analysis. Crit Care 2014; 18: 711.

48 Chu DK, Kim LH, Young PJ, et al. Mortality and morbidity in acutely ill adults treated with liberal versus conservative oxygen therapy (IOTA): a systematic review and meta-analysis. Lancet 2018; 391: 1693-1705.

49 Helmerhorst HJ, Schultz MJ, van der Voort PH, et al. Effectiveness and clinical outcomes of a two-step implementation of conservative oxygenation targets in critically ill patients: a before and after trial. Crit Care Med 2016; 44: 554-563.

50 The ICU-ROX Investigators and the Australian and New Zealand Intensive Care Society Clinical Trials Group. Conservative oxygen therapy during mechanical ventilation in the ICU. N Engl J Med 2020; 382: 989-998.

51 Barrot L, Asfar P, Mauny F, et al. Liberal or conservative oxygen therapy for acute respiratory distress syndrome. N Engl J Med 2020; 382: 999-1008.

52 Kelly RF, Hursey TL, Parrillo JE, et al. Effect of 100\% oxygen administration on infarct size and left ventricular function in a canine model of myocardial infarction and reperfusion. Am Heart J 1995; 130: 957-965.

53 Siemieniuk RAC, Chu DK, Kim LH, et al. Oxygen therapy for acutely ill medical patients: a clinical practice guideline. BMJ 2018; 363: k4169.

54 Angus DC. Oxygen therapy for the critically ill. N Engl J Med 2020; 382: 1054-1056.

55 Hofmann R, James SK, Jernberg T, et al. Oxygen therapy in suspected acute myocardial infarction. N Engl J Med 2017; 377: 1240-1249.

56 Gift AG, Stanik J, Karpenik J, et al. Oxygen saturation in postoperative patients at low risk for hypoxemia: is oxygen therapy needed? Anesth Anal 1995; 80: 368-372.

57 Pedersen T, Viby-Mogensen J, Ringsted C. Anaesthetic practice and postoperative pulmonary complications. Acta Anaesthesiol Scand 1992; 36: 812-818.

58 Mathew JP, Rosenbaum SH, O'Connor T, et al. Emergency tracheal intubation in the postoperative care unit: physician error or patient disease? Anesth Analg 1990; 71: 691-697.

59 Smetana GW, Lawrence VA, Cornell JE, et al. Preoperative pulmonary risk stratification for noncardiothoracic surgery: systematic review for the American College of Physicians. Ann Intern Med 2006; 144: 581-595.

60 Ramachandran SK, Thompson A, Pandit JJ, et al. Retrospective observational evaluation of postoperative oxygen saturation levels and associated postoperative respiratory complications and hospital resource utilization. PLoS One 2017; 12: e0175408.

61 Cirio S, Nava S. Pilot study of a new device to titrate oxygen flow in hypoxic patients on long-term oxygen therapy. Respir Care 2011; 56: 429-434.

62 Rice KL, Schmidt MF, Buan JS, et al. AccuO2 oximetry-driven oxygen-conserving device versus fixed-dose oxygen devices in stable COPD patients. Respir Care 2011; 56: 1901-1905.

63 L'Her E, N'Guyen QT, Pateau V, et al. Photoplethysmographic determination of the respiratory rate in acutely ill patients: validation of a new algorithm and implementation into a biomedical device. Ann Intensive Care 2019; 9: 11. 